\title{
Comparing Refractive and Scheimpflug-image Based Parameters between Right and Left Eyes and between Dominant and Non-dominant Eyes Related to Handedness
}

\author{
Noémi Tóth ${ }^{1}$, Adrienne Csutak ${ }^{1}$, Ziad Hassan $^{2}$, Eszter Deák ${ }^{1}$, Laszlo Modis Jr. ${ }^{1}$ \\ and Gabor Nemeth ${ }^{3^{*}}$ \\ ${ }^{1}$ Department of Ophthalmology, University of Debrecen, Debrecen, Hungary. \\ ${ }^{2}$ Orbi-Dent Refractive Surgery and Medical Center, Debrecen, Hungary. \\ ${ }^{3}$ Department of Ophthalmology, Borsod-Abaúj-Zemplén County Central Hospital and University \\ Teaching Hospital, Miskolc, Hungary.
}

Authors' contributions

This work was carried out in collaboration between all authors. Authors NT, ACs and GN designed the study. Author GN performed the statistical analysis. Authors ACs, ZH, ED wrote the protocol and authors NT, LM and GN wrote the first draft of the manuscript. Authors NT and ACs and GN managed the analyses of the study. Authors NT, LMJ and GN managed the literature searches. All authors read and approved the final manuscript.

Article Information

DOI: $10.9734 / O R / 2018 / 46026$ Editor(s):

(1) Dr. Ahmad M Mansour, Professor, Department of Ophthalmology, American University of Beirut, Lebanon.

(1) Anil Kumar Verma, Radhakrishnan Govt. Medical Coliege,

College, India.

(2) Tayo Julius Bogunjoko, Eye Foundation Hospital Group, Nigeria.

(3) Italo Giuffre, Catholic University of Roma, Italy. Complete Peer review History: http://www.sciencedomain.org/review-history/28013

Original Research Article

Received 08 October 2018

Accepted 15 December 2018

Published 31 December 2018

\section{ABSTRACT}

Aim: Our aim was to compare refractive and anatomical parameters between right and left eyes and between the dominant and non-dominant eyes of right- and left-handed patients.

Study Design: Clinical observational study.

Methodology: Refractometry, optical biometry and Scheimpflug photography were performed in 163, healthy subjects (138 right-handed, 25 left-handed). A hole-in-the-card test was used to determine sighting dominance. The parameters were compared in three groups: differences 
between the right and left eyes; differences between the dominant and non-dominant eyes; differences between the right-dominant and left-dominant eyes.

Results: The mean age of the patients was $46.42 \pm 20.60$ years. Among the right-handed patients, 77 were right-eye dominant and 61 were left-eye dominant. Significant differences were found regarding all observed parameters, excluding refraction data between the right and left eyes of the right-handed patients. Regarding dominant vs. non-dominant eyes of right-handed patients, only some data showed statistically significant differences. We could not find any significant differences in parameters of the left-handed group.

Conclusions: Significant differences were found in Scheimpflug-derived anatomical parameters of the left and right eyes of right-handed persons. However, when comparing the dominant and nondominant eyes, our results show only isolated differences. Sighthing dominance plays a role in ophthalmology, but this is probably not related to anatomical differences.

Keywords: Cornea; hand dominance; Scheimpflug imaging; sighthing dominance.

\section{INTRODUCTION}

The human body is essentially a basically bilateral, symmetrical structure. The left and right sides of the human body generally appear to be in bilateral symmetry, relative to the median vertical plane; for example, the eyes, ears and limbs. In 1903, Rosenbach claimed that most people have a dominant eye, even though each of their two eyes in isolation may provide equal vision and, in the case of unequal vision, the dominant eye is not always the eye with better visual acuity [1]. Moreover, the brain does not use the stimulus of light detected by the two eyes in equal ratio [2] and the visual performance was better using the dominant eye [3]. In general, ocular dominance is defined as the tendency to prefer visual input from one eye over the other [2] and may be further characterized as a status whereby one of the eyes commonly dominates or leads the other eye, both in attentional and in sensory aspects $[4,5]$.

Eye dominance, which is commonly determined with the easy to perform hole-in-the-card test $[6,7]$, provides the foundation for a range of clinical decisions, including contact lens correction, intraocular lens planning during cataract surgery and monovision adjusting, which is believed to be important in the control of reading ability and therefore patient satisfaction following monovision setting or treatment [8]. This clinical practice is based on the assumption that it will be easier to suppress blur in the nondominant eye than in the dominant one. Surgically induced anisometropia should not exceed 2.5 diopters (D), with the dominant eye generally being corrected for distance and the non-dominant eye for near vision. The concept of motor and sensory dominance has been developed over time yet it seems justified to state that both the importance and basis of eye dominance, whether motor or sensory, remains poorly understood.

Ocular dominance, especially sighting dominance plays an obvious role in the planning of ocular surgeries (primarily keratorefractive surgeries and different monovision settings) and may be present in the background of possible differences related to pain perception of the second eye surgery. Approximately 10 to $15 \%$ of the eyes' optical aberrations are higher-order aberrations, which account for some distinct visual quality-, dysphotopsia- and contrast sensitivity problems [9]. This, in addition to differences in common refractive errors, corneal higher order aberrations, especially coma aberrations, can be also interesting in the context of ocular dominance.

The question arises of whether, to some partial degree in the background of eye dominance, anatomical and/or refractive differences are present in addition to neurological causes. Our aim was to compare ophthalmological, measurable parameters (refractive and anatomical) between the right and left eyes, and between the dominant and non-dominant eyes of right- and left-handed patients.

\section{MATERIALS AND METHODS}

A total of 163 subjects, 138 with right-hand dominance and 25 with left-hand dominance were recruited at routine ophthalmological examinations, performed at the Ophthalmology Clinic, University of Debrecen, Hungary. To be included in the study, subjects were required to have normal anterior and posterior eye segment during a slit lamp examination. Patients did not wear contact lenses in the two days previous to the examinations. Subjects were excluded from the study if any of the following conditions 
existed: ocular surgery in the anamnesis, amblyopia, corneal pathologies or retinal diseases.

\subsection{Examinations}

Objective refractometry measurements were obtained using the Topcon KR8900 autorefractor (Topcon Co., Tokyo, Japan). These data refer to the total diopter value of the eye, i.e., the diopter value with which the refraction error could be corrected. Subjective refractive values (spherical $(\mathrm{S})$, cylindrical (C) and axis ( $\alpha$ ) of the cylinder) were measured using a trial frame. These subjective values refer to the diopter value of a glass, with which the spherical and cylindrical refractive errors could be improved. Objective and subjective refractometric measurements were performed without cycloplegia.

Keratometry readings and the magnitude and axis of the keratometric readings, and axial length were observed with IOLMaster (Carl Zeiss Meditec, Jena, Germany). All other anatomical parameters were measured with a Scheimpflug tomographer (Pentacam HR, Oculus Optikgeräte, Wetzlar, Germany, software version: 1.17r139). The following data were recorded with Pentacam HR: root-mean square (RMS) values of the lowerorder and higher-order aberrations and values of horizontal and vertical coma aberration on the anterior and posterior surfaces of the cornea.

\subsection{Sighting and Hand Dominance}

The hole-in-the-card test was employed to determine sighting dominance, which is one of several forms of ocular dominance. During the test, subjects were asked to hold a card with a 1 $\mathrm{cm}$ hole at the center with both their hands at arm's length. The subjects viewed a target six meters away through the hole, with both eyes open. Each eye was then covered in turn to identify the dominant one. When the dominant eye was covered, the target disappeared. When the non-dominant eye was covered, the target did not disappear. Each patient covered their eyes in turn, at least three times, until their response was sure about the dominant and nondominant eyes. Handedness was established with a clear decision by the patient about their dominant hand, based on which they use for writing and predominantly for performing other tasks.

\subsection{Data Analyses and Statistics}

Data were entered into a Microsoft Excel spreadsheet and statistical analyses were performed with the SPSS for Windows software (version 22.0, SPSS, Inc.). Means and standard deviations were used for data description. The data of right-handed and left-handed patients were separated into three data groups and further analysed. The first group was to test for differences between the right (RE) and left eyes (LE) of right-handed and left-handed people. The second was to test for differences between the dominant (DE) and non-dominant eyes (NDE) of right-handed and left-handed people. In the third test, differences were tested between the rightdominant eyes (RDE) and left-dominant eyes (LDE) of right-handed and left-handed people, i.e., differences between the data of right side dominant eyes and left side dominant eyes of right-handed or left-handed patients. The Wilcoxon rank-sum test was used for comparing all separated data groups. Two-way ANOVA was used to test interactions between two withinsubject factors (right/left eyes and eyedominance) on the dependent variables (refractive and anatomical parameters). The statistical significance level was set at $p<0.05$.

The research protocol adhered to the tenets of the Declaration of Helsinki and was approved by the Institutional Ethics Committee. Patients gave informed consent for participating in the present study.

\section{RESULTS}

The study included 163 patients (mean age $46.42 \pm 20.60$ years, range: $15.0-85.5$ years), of which 138 were right-handed and 25 were lefthanded. Among the right-handed patients, 77 were right-eye dominant and 61 were left-eye dominant. In the case of the left-handed patients, 13 were right-eye dominant and 12 were left-eye dominant. We noticed cross-dominance in $45.7 \%$ the patients, defined as the laterality of the hands and eyes being different, e.g., a right-handed person who was left-eye dominant. In all cases, the choices between the eyes and the hands were absolutely clear. The mean spherical equivalent refraction value of the patients was $0.47 \pm 2.2 \mathrm{D}$ (range: $-11.0 \mathrm{D}$ to $+5.0 \mathrm{D}$ ).

Refractive and Scheimpflug-image-derived anatomical parameters are presented in Table 1. Significant differences were found for all observed parameters, excluding spherical and 
cylindrical refraction data between the right and left eyes of right-handed patients. Regarding the dominant vs. non-dominant eyes of right-handed patients, RMS values of the corneal front surface, RMS LOA of the corneal front surface, RMS of the total cornea and RMS LOA of the total cornea showed a statistically significant difference. Regarding the right-dominant vs. leftdominant eyes, keratometry readings, axial length, some RMS data and horizontal coma aberration showed a statistically significant difference (Table 2).

We could not find any significant differences in the measured parameters of the left-handed study groups, separated as per the abovementioned three groups $(p>0.05$ in all parameters). Two-way ANOVA did not show any statistically significant interaction between the two factors (right/left eye and eye-dominance) on any of the dependent variables ( $p>0.05$ in all cases).

\section{DISCUSSION}

The objective of our study was to analyse the refractive and anatomical parameters of the anterior segment of the eye observed by a Scheimpflug-based device and to make observations on the differences in these data as a function of hand and eye dominance.

According to associations between eye and hand dominance, Pointer et al. [10] state that sighting dominance, handedness and visual acuity preferences are evidently not related at a level greater than statistical chance will predict, although the results in the literature is at least contradictory.

Some interesting literature data exists regarding the examination of possible differences between eyes in some measurable parameters. Statistically, higher accommodative amplitude was found in the dominant eye in young adults [11]. The relationship between ocular dominance and the macula was examined by Park et al. [12], who reported that ocular dominance was not an important determinant of human cone photoreceptor density at the fovea. Another interesting aspect is that pain scores were higher in dominant-side cataract surgery performed under topical anaesthesia [13]. Besides, Samarawickrama et al. [14] observed that in children, dominant eyes tended to be longer and more myopic than non-dominant eyes, but no ocular structural differences were observed between dominant and non-dominant eyes with the use of optical coherence tomography. We found prominent, strongly differences in Scheimpflug-derived anatomical parameters of the right and left eyes of right-handed persons.

Papers that comment on refractive differences between the two eyes exist. We could not find significant alterations in the refraction values of the different groups, which is consistent with a study conducted by Momeni-Moghaddam et al. [11]. Eser et al. [6] also did not find a spherical equivalent difference between eyes, which correlates with motor eye dominance in a large patient group. Conversely, Ito et al. [15] found that non-dominant eyes had greater myopic refractive errors and longer axial lengths compared to dominant eyes, especially in cases of high amounts of anisometropia. In this paper, the age of the examined group was different (a mean of 29 years) and the methodology was different (refractive error was determined with cycloplegic refraction), and the sample number was greater than in our work; we believe that these factors may have been present in the background of the differences. Linke et al. [16] analysed more than 10.000 myopic patients and found that the non-dominant eye of refractive surgery candidates was more myopic for the spherical equivalent anisometropia of more than $2.5 \mathrm{D}$, and more astigmatic for cylindrical anisometropia of more than $0.5 \mathrm{D}$. According to Mansour et al., right-handed people have 0.25 diopter of myopia more in the right eye than the left eye and left handed people have more myopia in the left eye by 0.25 diopter [17]. In this study, the refractive status of the population was different (a mean of $-4.0 \mathrm{D}$ ) and the size of the examined group was also significantly larger than in our paper, which is noted as a plausible reason for the different results found.

When comparing the right and the left eyes, as well as the right-dominant with the left-dominant eyes of right-handed persons, we found significant differences in the corneal radius of curvature and in the axial lengths, but failed to do so when comparing these parameters of dominant to non-dominant eyes. It is clear from our detailed data that in the case of keratometry readings, we found a 0.1 diopter difference between refraction values of the right and the left eyes. However, this difference was approximately 0.5-0.75 diopter between the rightdominant and the left-dominant eyes. The latter result is a truly and clinically significant difference. We found a $0.1 \mathrm{~mm}$ difference in axial lengths between the right-dominant and leftdominant eyes of right-handed patients. 
Table 1. Mean \pm standard deviations of refractive, keratometric, axial length, the lower- and higher-order aberration data of the right-handed patients

\begin{tabular}{|c|c|c|c|c|c|c|}
\hline & Right eyes & Left eyes & Dominant eyes & $\begin{array}{l}\text { Non-dominant } \\
\text { eyes }\end{array}$ & $\begin{array}{l}\text { Right-dominant } \\
\text { eyes }\end{array}$ & $\begin{array}{l}\text { Left-dominant } \\
\text { eyes }\end{array}$ \\
\hline Refr sph (D) & $-0.41 \pm 2.38$ & $-0.23 \pm 2.07$ & $-0.28 \pm 2.10$ & $-0.36 \pm 2.35$ & $-0.04 \pm 2.08$ & $-0.68 \pm 2.36$ \\
\hline Refr cyl (D) & $0.46 \pm 0.89$ & $0.47 \pm 0.87$ & $0.48 \pm 0.89$ & $0.45 \pm 0.87$ & $0.48 \pm 0.94$ & $0.45 \pm 0.81$ \\
\hline Refr axis $\left({ }^{\circ}\right)$ & $81.73 \pm 66.46$ & $68.59 \pm 66.21$ & $75.58 \pm 67.10$ & $74.74 \pm 66.22$ & $76.53 \pm 67.59$ & $73.43 \pm 65.44$ \\
\hline $\mathrm{K} 1$ (D) & $43.31 \pm 1.41$ & $43.41 \pm 1.42$ & $43.34 \pm 1.42$ & $43.38 \pm 1.40$ & $43.05 \pm 1.39$ & $43.75 \pm 1.33$ \\
\hline K2 (D) & $44.14 \pm 1.51$ & $44.25 \pm 1.42$ & $44.14 \pm 1.47$ & $44.23 \pm 1.48$ & $43.92 \pm 1.48$ & $44.53 \pm 1.34$ \\
\hline $\mathrm{AL}(\mathrm{mm})$ & $23.54 \pm 1.02$ & $23.48 \pm 1.00$ & $23.52 \pm 1.03$ & $23.50 \pm 1.00$ & $23.50 \pm 1.00$ & $23.40 \pm 0.97$ \\
\hline RMS CF $(\mu \mathrm{m})$ & $4.70 \pm 1.58$ & $5.06 \pm 4.67$ & $3.50 \pm 0.91$ & $3.58 \pm 0.94$ & $3.59 \pm 0.86$ & $5.19 \pm 4.93$ \\
\hline RMS HOA CF $(\mu \mathrm{m})$ & $1.31 \pm 0.46$ & $1.66 \pm 3.80$ & $1.13 \pm 0.20$ & $1.24 \pm 0.15$ & $1.18 \pm 0.17$ & $1.72 \pm 4.04$ \\
\hline RMS LOA CF $(\mu \mathrm{m})$ & $4.51 \pm 1.54$ & $4.70 \pm 2.88$ & $4.80 \pm 2.99$ & $4.39 \pm 1.28$ & $4.43 \pm 1.55$ & $4.80 \pm 2.99$ \\
\hline RMS CB $(\mu \mathrm{m})$ & $1.88 \pm 0.41$ & $1.91 \pm 0.47$ & $1.81 \pm 0.39$ & $1.72 \pm 0.33$ & $1.76 \pm 0.34$ & $1.98 \pm 0.42$ \\
\hline RMS HOA CB $(\mu \mathrm{m})$ & $0.48 \pm 0.26$ & $0.50 \pm 0.23$ & $0.39 \pm 0.022$ & $0.44 \pm 0.06$ & $0.41 \pm 0.048$ & $0.50 \pm 0.24$ \\
\hline RMS LOA CB $(\mu \mathrm{m})$ & $1.82 \pm 0.40$ & $1.84 \pm 0.44$ & $1.76 \pm 0.393$ & $1.66 \pm 0.33$ & $1.71 \pm 0.341$ & $1.91 \pm 0.37$ \\
\hline RMS Cornea $(\mu \mathrm{m})$ & $4.18 \pm 1.65$ & $4.56 \pm 5.00$ & $3.16 \pm 0.617$ & $3.36 \pm 0.92$ & $3.26 \pm 5.27$ & $4.60 \pm 5.27$ \\
\hline RMS HOA Cornea $(\mu \mathrm{m})$ & $1.25 \pm 0.47$ & $1.62 \pm 3.96$ & $1.18 \pm 0.18$ & $1.29 \pm 0.18$ & $0.24 \pm 0.18$ & $1.66 \pm 4.22$ \\
\hline RMS LOA Cornea $(\mu \mathrm{m})$ & $3.98 \pm 1.62$ & $4.18 \pm 3.16$ & $2.93 \pm 0.62$ & $3.01 \pm 0.95$ & $3.01 \pm 0.746$ & $4.20 \pm 3.28$ \\
\hline Z3 1 CF $(\mu \mathrm{m})$ & $-0.25 \pm 0.41$ & $0.31 \pm 0.70$ & $-0.35 \pm 0.202$ & $0.26 \pm 0.09$ & $-0.04 \pm 0.358$ & $0.05 \pm 0.80$ \\
\hline $\mathrm{Z3}-1 \mathrm{CF}(\mu \mathrm{m})$ & $-0.031 \pm 0.48$ & $-0.13 \pm 0.61$ & $-0.02 \pm 0.44$ & $-0.07 \pm 0.47$ & $-0.04 \pm 0.40$ & $0.00 \pm 0.63$ \\
\hline
\end{tabular}

Refr sph: refractive spherical value, Refr cyl: refractive cylindrical value, Refr axis: axis of refractive astigmatism.

K1: keratometric value at the flattest corneal meridian, K2: keratometric value at the steepest corneal meridian.

RMS: root mean square, CF: cornea Front, CB: cornea Back, HOA: higher-order aberration, LOA: lower-order aberration

Z3 1, Z3 -1: vertical and horizontal components of the corneal coma aberration. 
Table 2. P values of compared parameters of right vs. left eyes, dominant vs. non-dominant eyes and right-dominant vs. left-dominant eyes of the right-handed patients

\begin{tabular}{|c|c|c|c|}
\hline & $\begin{array}{l}\text { Right vs. left } \\
\text { eyes }\end{array}$ & $\begin{array}{l}\text { Dominant vs. non- } \\
\text { dominant eyes }\end{array}$ & $\begin{array}{l}\text { Right-dominant vs. left- } \\
\text { dominant eyes }\end{array}$ \\
\hline Refr sph (D) & 0.218 & 0.724 & 0.159 \\
\hline Refr cyl (D) & 0.767 & 0.901 & 0.74 \\
\hline Refr axis $\left({ }^{\circ}\right)$ & $0.012^{*}$ & 0.608 & 0.672 \\
\hline K1 (D) & $0.01^{*}$ & 0.603 & $0.001^{*}$ \\
\hline K2 (D) & $0.015^{*}$ & 0.084 & $0.005^{x}$ \\
\hline $\mathrm{AL}(\mathrm{mm})$ & $0.006^{x}$ & 0.856 & $0.02^{*}$ \\
\hline RMS CF $(\mu \mathrm{m})$ & $<0.001^{*}$ & $0.019^{\pi}$ & $0.035^{\pi}$ \\
\hline RMS HOA CF $(\mu \mathrm{m})$ & $<0.001^{*}$ & 0.67 & 0.381 \\
\hline RMS LOA CF $(\mu \mathrm{m})$ & $<0.001^{*}$ & $0.018^{x}$ & $0.03^{x}$ \\
\hline RMS CB $(\mu \mathrm{m})$ & $<0.001^{*}$ & 0.263 & $0.012^{x}$ \\
\hline RMS HOA CB $(\mu \mathrm{m})$ & $<0.001^{*}$ & 0.827 & 0.621 \\
\hline RMS LOA CB $(\mu \mathrm{m})$ & $<0.001^{*}$ & 0.157 & $0.008^{*}$ \\
\hline RMS Cornea $(\mu \mathrm{m})$ & $<0.001^{*}$ & $0.044^{*}$ & 0.128 \\
\hline RMS HOA Cornea $(\mu \mathrm{m})$ & $<0.001^{*}$ & 0.919 & 0.678 \\
\hline RMS LOA Cornea $(\mu \mathrm{m})$ & $<0.001^{*}$ & $0.046^{*}$ & 0.129 \\
\hline Z3 $1 \mathrm{CF}(\mu \mathrm{m})$ & $<0.001^{*}$ & 0.787 & 0.596 \\
\hline $\mathrm{Z} 3-1 \mathrm{CF}(\mu \mathrm{m})$ & $<0.001^{*}$ & 0.612 & $0.009^{x}$ \\
\hline
\end{tabular}

Refr sph: refractive spherical value, Refr cyl: refractive cylindrical value, Refr axis: axis of refractive astigmatism. K1: keratometric value at the flattest corneal meridian, K2: keratometric value at the steepest corneal meridian. AL: axial length; RMS: root mean square, CF: cornea Front, CB: cornea Back, HOA: higher-order aberration, LOA: lower-order aberration; Z3 1, Z3 -1: vertical and horizontal components of the corneal coma aberration. : statistically significant differences, i.e. $p$ value $<0.05$ between data groups.

Regarding dominant vs. non-dominant eyes, it looks as though mostly neurophysiological reasons may be in the background of the differences, rather than anatomical reasons, as it was previously suggested [3]; now, we don't have any other explanation for these differences. According to our results, we showed only a few statistically significant, isolated differences in the anatomical parameters, when comparing the dominant and non-dominant eyes. The results of Chaumillon et al. [18] demonstrated the influence of the eye dominance in the processing of visual information for the production of hand movements in right-handed patients. Moreover, their findings demonstrated a clear impact of eye dominance on neural mechanisms involved in converting visual inputs into motor commands. They stated that the effect of eye dominance strongly depended on whether the patients were right- or left-handed [18].

The overwhelming majority of higher-order aberrations are of corneal origin [19]. Zernike polynomials are used for the mathematical deduction of higher-order aberrations, which have been known for more than 50 years [20,21]. Among the third- and fourth-order higher order aberrations, spherical aberrations and coma-like deviations are the most crucial, since these cause the most frequent complaints following refractive surgery. These aberrations are often the cause of complaints of blurry vision, dysphotopsia or monocular diplopia. In the case of right-handed people, we also found significant differences in the anterior and posterior surfaces of right and left eyes with respect to lower- and higher-order corneal aberrations, while the differences were mostly not significant when the dominant to non-dominant eyes or right-dominant to left-dominant eyes were compared.

Coma aberration appears when rays entering the eye are inclined at an angle to the optical axis. Rays entering at the sides intersect the focal surface at different altitudes than those that enter in the middle. This results in an asymmetrical distribution of an image from a point source, giving it an elongated, comet-like shape. Coma aberrations may have a role in aiding reading skills, according to literature data [21,22]. Regarding coma aberrations, we found highly significant differences between the right and left eyes of right-handed patients, specifically, higher RMS values in the case of left eyes. This difference was also significant in cases of RMS values of LOA and HOA on the anterior and posterior surfaces of the cornea, and on the entire cornea. 
Our examinations had some limitations. First, we were unable to find any significant differences in the measured parameters of the left-handed study groups, although that may have been due to the low number of studied left-handed volunteers, meaning we were unable to gain reliable comparison data for the right and left eyes or dominant vs. non-dominant eyes of lefthanded persons. Another limitation was that the parameters we observed were arbitrarily chosen, i.e., other measured parameters may also prove interesting for such a comparison (e.g., anterior chamber parameters, corneal thickness, elevation data, and single aberration data).

\section{CONCLUSION}

Prominent differences were found in Scheimpflug-derived anatomical parameters of the right and left eyes of right-handed persons. However, when comparing the dominant and non-dominant eyes, our results show only a few statistically significant, isolated differences in these parameters, without any prominent differences. Ocular dominance, especially sighting dominance plays a role in planning certain ocular surgeries, establishing monovision settings and possible differences in terms of pain experienced at the second eye surgery; however, the factors are likely not related to the discussed anatomical differences. Further examinations are required to seek out the clinical relevance and explanation of significant differences between parameters of the right and left eyes of righthanded patients.

\section{CONSENT}

Patients gave informed consent for participating in the present study.

\section{ETHICAL APPROVAL}

The research protocol adhered to the tenets of the Declaration of Helsinki and was approved by the Institutional Ethics Committee.

\section{COMPETING INTERESTS}

Authors have declared that no competing interests exist.

\section{REFERENCES}

1. Rosenbach $O$. Ueber monokulare vorherrschaft beim binokularen Sehen.
Münchener Medizinische Wochenschrift 1903;30:1290-2.

2. Porac C, Coren S: The dominant eye. Psychol Bull. 1976;83(5):880-97.

3. Shneor E, Hochstein S. Eye dominance effects in feature search. Vision Res. 2006;46(25):4258-69.

4. Roth HL, Lora AN, Heilmann KM. Effects of monocular viewing and eye dominance on spatial attention. Brain. 2002;125(Pt 9):2023-5.

5. Suttle C, Alexander J, Liu M, Ng S, Poon J, Tran T. Sensory ocular dominance based on resolution acuity, contrast sensitivity and alignment sensitivity. Clin Exp Optom. 2009;92:2e8.

6. Eser I, Durrie DS, Schwendeman F, Stahl JE. Association between ocular dominance and refraction. J Refract Surg. 2008; 24(7):685-9.

7. Lopes-Ferreira D, Neves $H$, Queiros A, Faria-Ribeiro M, Peixoto-de-Matos SC, Gonzalez-Meijome JM. Ocular dominance and visual function testing. Biomed Res Int. 2013;2013:238943.

8. Seijas O, Gomez de Liano P, Gomez De Liano R, Roberts CJ, Piedrahita E, Diaz E. Ocular dominance diagnosis and its influence in monovision. Am J Ophthalmol. 2007;144(2):209e16.

9. Fan-Paul NI, Li J, Miller JS, Florakis GJ. Night vision disturbances after corneal refractive surgery. Surv Ophthalmol. 2002;47(6):533-46.

10. Pointer JS. Sighting dominance, handedness, and visual acuity preference: three mutually exclusive modalities? Ophthalmic Physiol Opt. 2001;21(2):11726.

11. Momeni-Moghaddam $\mathrm{H}$, McAlinden $\mathrm{C}$, Azimi A, Sobhani M, Skiadaresi E. Comparing accommodative function between the dominant and non-dominant eye. Graefes Arch Clin Exp Ophthalmol. 2014;252(3):509-14.

12. Park SP, Chung JK, Greenstein V, Tsang $\mathrm{SH}$, Chang S. A study of factors affecting the human cone photoreceptor density measured by adaptive optics scanning laser ophthalmoscope. Exp Eye Res. 2013;108:1e9.

13. Aslankurt M, Aslan L, Başkan AM, Aksoy A, Silay E, Yıldız H. Pain and cooperation in patients having dominant-side or nondominant-side phacoemulsification. J Cataract Refract Surg. 2014;40(2):199202. 
14. Samarawickrama C, Wang JJ, Huynh SC, Wang XY, Burlutsky G, Stapleton F et al. Macular thickness, retinal thickness, and optic disk parameters in dominant compared with nondominant eyes. J AAPOS. 2009;13(2):142-7.

15. Ito M, Shimizu K, Kawamorita T, Ishikawa $\mathrm{H}$, Sunaga K, Komatsu M. Association between ocular dominance and refractive asymmetry. J Refract Surg. 2013; 29(10):716e20.

16. Linke SJ, Baviera J, Munzer G, Steinberg J, Richard G, Katz T. Association between ocular dominance and spherical/astigmatic anisometropia, age, and sex: Analysis of 10,264 myopic individuals. Invest Ophthalmol Vis Sci. 2011;52(12):9166-73.

17. Mansour AM, Sbeity Z. Refraction and Hand dominance. Acta Ophthalmol Scand. 2003;81(1):82-3.
18. Chaumillon R, Blouin J, Guillaume A. Eye dominance influences triggering action: The Poffenberger paradigm revisited. Cortex. 2014;58:86-98.

19. Artal P, Guirao A, Berrio E, Williams DR. Compensation of corneal aberrations by the internal optics in the human eye. J Vis. 2001;1(1):1-8.

20. Ivanof $A$. About the spherical aberration of the eye. J Opt Soc Am. 1953;46(10):901-3.

21. Nanavaty MA, Spalton DJ, Marshall J. Effect of intraocular lens asphericity on vertical coma aberration. J Cataract Refract Surg. 2010;36(2):215-21.

22. de Gracia P, Dorronsoro C, Gambra E, Marin $G$, Hernández $M$, Marcos $S$. Combining coma with astigmatism can improve retinal image over astigmatism alone. Vision Res. 2010; 50:2008-14.

(c) 2018 Tóth et al.; This is an Open Access article distributed under the terms of the Creative Commons Attribution License (http://creativecommons.org/licenses/by/4.0), which permits unrestricted use, distribution, and reproduction in any medium, provided the original work is properly cited.

Peer-review history:

The peer review history for this paper can be accessed here: http://www.sciencedomain.org/review-history/28013 